\title{
Una aproximación lexicográfica a los insultos de grupo en el español del Uruguay
}

\author{
A lexicographic approach to slurs in Uruguayan \\ Spanish
}

\author{
Ana Clara Polakof (iD Andrés de Azevedo \\ Departamento de Teoría del Lenguaje y Lingüística, Universidad de la República \\ / Sistema Nacional de Investigadores, Uruguay
}

anaclarapo@gmail.com

aazevedo@vera.com.uy

\section{G ACCESO ABIERTO / OPEN ACCESS}

Cita: Polakof, Ana Clara, de Azevedo, Andrés (2020). Una aproximación lexicográfica a los insultos de grupo en el español de Uruguay. Textos en Proceso, 6(1), pp. 146-159.

https://doi.org/10.17710/tep.2020.6. 1.8polakofdeazevedo

Editores: Esperanza Alcaide Lara, Universidad de Sevilla, Ana Pano Alamán, Università di Bologna

Recibido: 29-03-2020

Aceptado: 25-05-2020

Conflicto de intereses: Los autores han declarado que no poseen conflicto de intereses.

Copyright: @ A Ana Clara Polakof; Andrés de Azevedo. Esta obra está bajo licencia Creative Commons Reconocimiento 4.0.

\section{Resumen}

El presente trabajo tiene por cometido incursionar en el estudio de los insultos de grupo -expresiones lingüísticas utilizadas para expresar una actitud peyorativa hacia una persona como integrante de un colectivo- desde un abordaje lexicográfico. Tal perspectiva toma algunos posicionamientos y planteos del debate actual sobre los insultos de grupo provenientes de la filosofía del lenguaje con el fin de iluminar sus posibles consecuencias en la confección y análisis de los diccionarios. Sobre la base de Pullum (2018), y el empleo de la distinción entre información semántica y metainformación, se estudia en particular el Diccionario del Español del Uruguay (DEU, 2011) en cuanto a la presentación de seis entradas léxicas correspondientes a insultos de grupo relativos a las clases sociales. Los resultados primarios obtenidos revelan un manejo no homogéneo de los criterios para diferenciar el contenido netamente semántico de otras informaciones pertinentes más ligadas al uso efectivo y dependiente del contexto. El trabajo, inscripto en un enfoque interdisciplinario, pauta un primer paso en el estudio lexicográfico y lingüístico de los insultos de grupo, tema muy poco estudiado para el español.

Palabras clave: insultos de grupo, lexicografía, filosofía del lenguaje, semántica.

\section{Abstract \\ The goal of this paper is to explore the analysis of slurs (linguistic expressions which are used to express a pejorative attitude towards a person as a part of a group) from a lexicographic approach. Such an approach takes into account some of the philosophy of language current debates with respect to slurs in order to enlighten its}


possible consequences in the elaboration and analysis of dictionaries. Based on Pullum (2018), and his appropriation of the distinction between semantic information and metadata, we analyze the Diccionario del Español del Uruguay (DEU, 2011) with regards to six lexical entries which correspond to social classes slurs. The primary results show a non-homogeneous management of the criteria to differentiate net semantic content from other relevant information which are linked to the actual use and depend upon the context of the lexical item. This paper, which is an outcome of an interdisciplinary effort to understand the use of slurs, marks a first step towards de lexicographic and linguistic study of slurs, an issue in Spanish that has not received much attention yet.

Keywords: slurs, lexicography, philosophy of language, semantics.

\section{Introducción}

Los insultos de grupo son expresiones lingüísticas utilizadas para expresar una actitud peyorativa hacia una persona como integrante de un colectivo. Pueden clasificarse según su origen (gringo, bolita, canario, gallego), su raza (negro), su orientación sexual (puto, mariposón), su etnia o religión (judaca), su ideología (facho, nazi, anarco, etc.), modo de vida (puta). Son, a su vez, expresiones que pueden no alterar el valor veritativo-funcional de una oración, por lo que son propicios para estudiar los límites entre semántica y pragmática. Asimismo, se prestan para ser considerados desde una perspectiva interdisciplinaria que tome en cuenta, por un lado, aspectos propiamente lingüísticos (como ser su modo de construcción, características de su empleo, peculiaridades de su codificación, etc.) y por otro, asuntos de naturaleza filosófica vinculados a su caracterización y utilización (como ser el lugar que deben ocupar en la semántica veritativofuncional, si deben o no tener extensión, etc.).

Este trabajo en proceso, que es el resultado de un esfuerzo interdisciplinario, busca dar una cobertura lingüística a un problema que es de fondo filosófico. ${ }^{1}$ Tiene como objetivo la aproximación a una de las tantas aristas lingüísticas que el tema convoca, y que guarda relación con el abordaje lexicográfico, es decir, el comprendido en la elaboración y análisis crítico de los diccionarios.

Seguiremos a Pullum (2018), quien hizo una propuesta de reacomodación de las entradas para el American Heritage Dictionary del inglés. Intentaremos una aproximación similar con el Diccionario del Español del Uruguay (DEU, 2011). Este diccionario conforma un corpus del léxico del habla uruguaya que tiene como particularidades el incorporar voces en uso desde comienzos del siglo XX y el de no ser un "diccionario de uruguayismos" (DEU, 2011, p. 15), en el sentido de que contempla junto a los términos exclusivos del país, aquellos que son compartidos en la región. Su propósito es recoger la variedad rioplatense del español en sus formas típicas o peculiares.

En la próxima sección, haremos una presentación de algunas de las propuestas que existen en el ámbito de la filosofía del lenguaje para dar cuenta de la interpretación de los insultos de grupo. Después, presentaremos el trabajo de

\footnotetext{
${ }^{1}$ Con tal fin, conformamos un grupo interdisciplinario con una propuesta de trabajo - Una aproximación interdisciplinaria a los insultos de grupo en Uruguay" - que fue financiada en 2018 por el programa Semillero de Iniciativas Interdisciplinarias (Espacio Interdisciplinario, UdelaR).
} 
Pullum (2018) al que tomaremos como modelo para trabajar con el español del Uruguay. Describiremos, en esa sección, las características del DEU (2011). A continuación, presentaremos nuestro análisis y discutiremos qué ventajas podría tener para un tratamiento de los insultos de grupo la reescritura que en esta sección proponemos. Finalmente, haremos unas breves consideraciones de cierre.

\section{Marco teórico: los insultos de grupo en la filosofía del lenguaje}

Los insultos de grupo han venido siendo estudiados en las últimas décadas principalmente por filósofos del lenguaje y como una forma de acercarse y contribuir al análisis del discurso de odio (término originado en las décadas de los 50 y los 60 en Estados Unidos) que surge a partir de una profunda revisión del marco de la virtud cívica (Nunberg, 2018). ${ }^{2}$ La consideración propiamente lingüística de los insultos de grupo es un campo que ha recibido una atención comparativamente menor. Dada esta situación no ha de extrañar que sean muy escasos los estudios referidos al comportamiento de estos términos en el español (una excepción es Orlando y Saab, 2019 y en prensa). Debido al interés casi exclusivamente filosófico que han tenido, el problema que se ha tendido a considerar es establecer cómo afecta el valor de verdad de una proposición la utilización de un insulto de grupo, como en $(1)^{3}$ :

1) ... tú, tú y nadie más que tú. No seas el sudaca subdesarrollado de tu propio universo, no hagas honor a lo peor de tu cultura.

La pregunta filosófica que surge, a partir de ejemplos como el anterior, es ¿puede ser verdadera una afirmación como la anterior? Si admitiéramos que los sudacas existen, (1) sería verdadera. De ser así, seríamos racistas porque admitiríamos que existe una clase de humanos proveniente de América Latina que es inferior a la clase de humanos proveniente de Europa. Sin embargo, la mayoría de los filósofos del lenguaje parecen defender que, para el caso, los sudacas no existen (Nunberg, 2018; Croom, 2013; Neufeld, 2019, entre otros). Ahora, si no existen los sudacas, ¿cuál es el valor de verdad de (1)? Para intentar responder esta pregunta, surgen varias teorías y marcos teóricos en lo que respecta al tratamiento de los insultos de grupo.

El problema se reduce, entonces, a explicar qué papel está jugando el insulto de grupo sudaca en dicha proposición. Hay dos posibles salidas para tratar tales insultos (Sosa, 2018): una semántica y una no semántica. La primera debe dar cuenta del valor de verdad de las proposiciones. Una opción dentro de la primera alternativa sería tratar el sintagma definido como un nombre vacio y defender que no refiere. Es decir, que no tiene extensión. Esto es algo que defiende Hom (2012).

\footnotetext{
${ }^{2}$ Así, por ejemplo, Cepollaro y Zeman (2020, p.1) señalan que los insultos de grupo son el caso más prototípico de discurso de odio. La bibliografía sobre insultos de grupo desde el punto de vista de la filosofía del lenguaje es amplísima. Para no abrumar con referencia a autores, nos limitamos a señalar que los posicionamientos principales pasan por estudiar el contenido derogatorio de tales términos con relación a las condiciones de verdad, los estereotipos, las presuposiciones, las implicaturas convencionales y conversaciones, los actos de habla, las reglas de uso, las inferencias pragmáticas generales y la prohibición. Por una enumeración que incluye referencias a los diversos autores ver Cepollaro (2017) y Nunberg (2018). Sobre discurso de odio ver Matsuda (1993) y Maitra \& McGowan (2012).

${ }^{3}$ Los ejemplos numerados del español (1), (2) y del (5) al (11) fueron tomados del Corpus del Español (Davies) en https://www.corpusdelespanol.org/web-dial/ (último acceso: 28 de mayo de 2020).
} 
Podríamos, también, proporcionar una salida clásica inspirada en Russell (1905): dado que no hay sudacas, cualquier proposición que incluya dicho sustantivo será falsa. Sin embargo, pareciera ser que en la afirmación anterior hay una persona de la cual se está hablando que cumpliría con el requisito de ser sudaca. Por lo que pudiera llegar a ser referencial. La segunda salida establece que no es la palabra la que discrimina, sino que es su uso lo que hace que sea un acto discriminatorio (Nunberg, 2018). Esta posición se centra en el uso del insulto y no en el valor de verdad de la proposición. Sin embargo, ello no la exime de procurar clarificar qué valores de verdad le caben a la proposición en cuestión. Esta aproximación debe, también, dar cuenta de cómo discriminan los insultos de grupo si ese significado peyorativo no está en la semántica del ítem léxico.

Una respuesta no-semántica de los insultos de grupo suele involucrar la idea de que estos tienen una contraparte neutra ${ }^{4}$. Dicha contraparte provee las condiciones veritativo-funcionales para que la afirmación sea verdadera, pero no las condiciones de uso que establecen que al emplear dicho ítem se esté discriminando. El ejemplo (1) podría transformarse en el ejemplo (2) que contiene un término neutro:

2) No seas el sudamericano subdesarrollado de tu propio universo.

En (2) sudaca es substituido por un término que por su carácter no se presta para un uso discriminatorio. El término neutro informa aquí la zona geográfica en el que la persona ha nacido.

Hay varias aproximaciones a este problema (ver Anderson y Lepore, 2013; Hom, 2012; McCready, 2010; Nunberg, 2018; Potts, 2005; Sosa, 2018; Whiting, 2013; Williamson, 2009, entre otros) ${ }^{5}$. Sin embargo, debido al perfil lingüístico de este trabajo, nos centraremos en la que parece describir de mejor manera las intuiciones acerca del nivel lingüístico en el que se da la discriminación. Consideramos que la línea planteada por Nunberg (2018) y seguida por Pullum (2018) es la que mejor da cuenta de ello. Dicha propuesta hace uso de las implicaturas conversacionales de Grice (1975) para explicar cómo afirmaciones del tipo (1) pueden ser discriminatorias. Propone que los efectos discriminatorios que provoca el uso de insultos de grupo -como sudaca, bolita, gallego, etc.- se deben a la explotación de la Máxima de Manera o de algún otro principio conversacional. Es posible pensar que la implicatura se produce al usar una submáxima de la forma: "Use appropriate language" (Nunberg, 2018, p. 244). Implica que hagamos decisiones adecuadas cuando utilizamos la lengua en distintas situaciones comunicativas que dependen de las normas sociales vigentes. Si dichas máximas (o submáximas) no son consideradas, las implicaturas surgen ${ }^{6}$. Nunberg considera que la diferencia entre redskin (piel roja) e indian (indio) está en la prescripción de las convenciones de un grupo cuyos miembros tienen actitudes derogatorias sobre los indios americanos. Las implicaciones negativas al usar el término peyorativo surgen gracias a la asociación con el discurso de un cierto tipo de grupo. En sus propias

\footnotetext{
${ }^{4}$ La noción de contraparte neutra desempeña un papel importante en muchos de los enfoques filosóficos sobre los insultos, ya sea como parte de la definición o como un rasgo característico (cf. DiFranco, 2015, p. 29; Nunberg, 2018, p. 262 y Orlando y Saab, 2019).

${ }^{5}$ Por críticas a esta aproximación, ver DiFranco (2015).

${ }^{6}$ Aunque esta propuesta presenta ciertos problemas (ver Neufeld, 2019) tiene la ventaja de dar cuenta de los datos y por ello nuestra preferencia por ella en este trabajo.
} 
palabras: "In a nutshell: racists don't use slurs because they're derogative; slurs are derogative because they're the words that racists use" (Nunberg, 2018, p. 244).

Los insultos no difaman o menosprecian en o por sí mismos, sino por sus asociaciones. Esto se traduce en la idea de que, aun teniendo un cierto contenido semántico, lo que los vuelve insultos es un tipo de información suplementaria. Nunberg señala que "there are a lot of things we know about words which affect the way we use them but which are all the same not themselves part of its meaning." (ibid., p. 264). Sus ejemplos incluyen información relativa a procedencia social y geográfica, frecuencia (corriente, arcaico), género/estilo discursivo (formal, coloquial), ámbito de uso (botánica, etc.) y efecto u recepción característica (humorístico, etc.) (op. cit., 265). Como puede verse, se trata de un vasto dominio en el que ingresan aspectos sociolingüísticos y diversos tipos de efectos dependientes de la emisión efectiva y del contexto. En la misma línea, Pullman refiere a cuestiones de tono y estilo, rasgos estéticos, efectos sociales de las formas de tratamiento honoríficas, vulgaridad o trato educado, ofensividad o grosería, etc. (Pullman, 2018, p.170). Se trata de "additional facts that do not form part of the inherent meaning of the expression but may influence pragmatic interpretation or have other effects on the impact of an utterance in context" (ibid. pp. 169-170).

Nunberg (2018) propone que se debe separar el significado de la metainformación. El primero aporta las condiciones veritativo-funcionales. La segunda brinda información acerca de los usos y convenciones de un determinado ítem léxico y no pertenece a su significado convencional. Se entiende así que no es parte del significado convencional de sudaca que sea discriminatorio respecto de los sudamericanos. Sin embargo, esa metainformación puede dar lugar a las implicaturas conversacionales mencionadas.

Es interesante notar que este tipo de implicatura no se da solo con los insultos de grupo, sino también con el léxico en proceso de cambio. Por ejemplo, el uso de propio en el habla joven del español uruguayo (Oggiani y Bértola, 2019) puede ser visto como vulgar por una persona mayor a 50 años. Sin embargo, que lo sea no es parte de su significado. Al igual que los insultos de grupo, puede dar lugar a algún tipo de implicatura. En el ejemplo (3) se presenta una afirmación en la que el hablante entiende que se compró la mejor campera:

3) La propia campera me compré (Oggiani y Bértola, 2019, p. 63).

Aunque se podría pensar que la metainformación constatada en (3) -el rasgo de vulgaridad que comporta para ciertos hablantes- pertenece a la semántica de la expresión, no es el caso. A nivel semántico propio contiene únicamente el rasgo de valoración superlativa. La metainformación, entre tanto, nos permite hacer implicaturas del tipo: quien usa la afirmación pertenece a determinado grupo social que difiere del nuestro. Lo mismo puede ser dicho de los insultos de grupo, y esto es lo que defiende Nunberg (2018).

Nunberg (op. cit., pp. 266-270) argumenta que el uso de los insultos da lugar a una "maniobra conversacional" mediante la cual el hablante, al emplear de forma deliberada una expresión no habitual (en el sentido de que no sería la normalmente utilizada en ese contexto) -el insulto-, se asocia con las actitudes con de un grupo cuyas normas se alejan de las opciones lingüísticas habituales que gobernarían la situación discursiva. Así, cuando un hablante usa sudaca en lugar de sudamericano, está haciendo caso omiso (voluntariamente) de la convención que estipula que debe 
utilizarse el segundo y no el primero. De esta manera, pauta su afiliación a un grupo con actitudes heterodoxas respecto de los sudamericanos (o el grupo que sea) y las reafirma. Nunberg denomina "ventrilocuismo" a este uso lingüístico. Las implicaturas a que da lugar - "implicaturas ventriloquísticas"- son un tipo de implicatura conversacional derivadas de la Máxima de Manera de Grice. Entre los rasgos que las caracterizan están el de ser difíciles de cancelar; el de soler ser activadas por el uso de palabras que se alejan de la norma habitual que rige la conversación, y el de estar orientadas hacia el hablante (el uso deliberado de una expresión alternativa evoca la actitud o punto de vista implícito en la palabra) y no hacia las condiciones de verdad del enunciado.

Para este autor el ventrilocuismo comportado por el uso de insultos de grupo se corresponde con un tipo particular de acto de habla, el "afiliatorio" (Nunberg, op. cit., p. 273), y en la variedad específica correspondiente al uso de términos que denotan una categoría socialmente controversial.

Como se desprende de lo anterior, para que estos actos de habla sean posible debe existir un término default ${ }^{7}$, normal, que está siendo rechazado en favor de la desviación de la norma. Por lo tanto, sudaca es un insulto de grupo porque existe el término default sudamericano que tiene su misma extensión. Tal término tiene que existir para que se reconozca a sudaca como un insulto de grupo.

El punto de vista por el que Nunberg aboga es que "slurring is just a special case of the way speakers exploit sociolinguistic variation to create self-presentations and invest their utterances with attitude" (op. cit., p. 290). Si tenemos en consideración las implicaturas que se pueden hacer en la utilización de palabras como propio, y cómo funciona la lengua en sociedad, la postura de Nunberg parece ser más que viable.

A continuación, presentamos la metodología basada en el trabajo de Pullum (2018).

\section{Metodología: Pullum (2018) y el DEU (2011)}

Nuestra inspiración, y referencia más importante, la constituye el trabajo de Pullum (2018), que incorpora al estudio de los insultos de grupo una consideración lexicográfica. La idea básica es que una entrada lexicográfica no debería confundir la información semántica con la información extra-semántica.

Luego de analizar el diccionario de lengua inglesa The American Heritage Dictionary (AHD) Pullum concluye que no hay un tratamiento homogéneo de los insultos, al haber diferencias en cómo se los define, lo que evidencia a su turno falta de importantes diferenciaciones conceptuales (como la que hay entre usos literales y no literales, y la distancia que media entre estos últimos y los insultos).

Pullum propone que el diccionario debería diferenciar entre la información semántica y, lo que hemos venido trabajando en términos de metainformación (metadata en Nunberg, 2018), ese cúmulo de información o conocimiento asociado a una palabra y que forma parte de lo que los hablantes competentes saben a propósito del término y su uso.

The fact is that the derogatory aspects of the conveyed effect of slurs, the part of them that we do not like or approve of, should be covered not in the specification

\footnotetext{
7 Este término se corresponde en gran medida con la "contraparte neutra" a la que hacíamos mención más arriba. Se trata de una expresión con similar denotación, pero libre de la dimensión evaluativa del insulto. Nunberg (2018) no se contenta con esta equiparación por cuanto entiende que la neutralidad no es simplemente de tipo semántico sino más bien cultural.
} 
of literal meaning, which is what the $A H D$ seems to be trying to do (while guarding itself against being accused of voicing the derogatory associated attitudes), but —as Nunberg (2015) proposes - in the metadata associated with the word. (Pullum, 2016, p. 185)

Por cierto que diversos tipos de metainformación son constitutivos de cualquier entrada léxica de un diccionario. Piénsese en la información gramatical (nombre, adjetivo, género, etc.). Cuando no se trata de este tipo suele acotarse a aquellos rasgos de las palabras que se apartan de las normas de la lengua formal y que gobiernan el lenguaje del propio diccionario (Nunberg, 2018). Allí aparecen cosas como coloquial, formal, humorístico, o como veremos, espontáneo, vulgar, despectivo).

La propuesta consiste en ampliar el rango de la metainformación para incorporar cuestiones léxicas, con información social (o sociolingüística) y pragmática, proporcionando así el contexto relevante para poder entender un insulto de grupo.

Mostramos, en (4), el ejemplo que toma Pullum del AHD y, en (5), su propuesta:

4) dyke n. Offensive Slang Used as a disparaging term for a lesbian.

5) dyke n. Semantics: lesbian. Metadata: slang, generally disparaging or offensive.

Pullum interpreta que expresiones como "used as" ("usado como"), "considered to be" ("se considera") y "regarded as" ("es visto como") pautan el incumplimiento básico de un diccionario que es el de aportar el significado de una palabra. Lo que se ofrece, en cambio, es un reemplazo mediante una observación metalingüística acerca de para qué se usa, o, una afirmación que compete a la sociedad en vez de la definición semántica directa. Una entrada correcta de un insulto ha de contener un contenido descriptivo, no un rodeo. (5), a diferencia de (4), propone un tratamiento diferenciado de la semántica y la metainformación léxica.

En este trabajo intentaremos aplicar la propuesta de Pullum a algunos ítems léxicos seleccionados sobre la base de su frecuencia de aparición en el corpus Davies, a partir de un previo análisis del DEU. Utilizamos el corpus para ver si efectivamente se trata de insultos de grupo que se usan en nuestra variedad. ${ }^{8}$

A diferencia de lo que ocurre en AHD, donde se recurre con frecuencia a las referidas fórmulas parafrásticas que ilustramos en (4), el DEU parecería optar por dar directamente las definiciones. Aún así tiene ciertas características que merecen una descripción. En primer lugar, introduce las previsibles marcas gramaticales que permiten establecer la categoría gramatical a la que pertenece la palabra, así como información de género (para sustantivos y adjetivos), de transitividad (para el caso de los verbos), entre otras. En segundo lugar, aparecen una serie de marcas que procuran contemplar otro tipo de información juzgada pertinente relativa a los términos. "Además de las marcas gramaticales, cada acepción cuenta con otras que proporcionan información sobre la frecuencia y la extensión de uso, los registros,

\footnotetext{
${ }^{8}$ Hay insultos de grupo que registramos en el DEU, pero que no tienen ningún registro en el Corpus del Español: Web/Dialects de Davies. Algunos ejemplos son: boniatero (insulto referido a la localidad de origen del individuo), calepino (insulto referido al partido político al que pertenece), calotero (insulto referido a modo de vida), entre otros. Los insultos que elegimos tienen registros en dicho corpus.
} 
los niveles lingüísticos, etc.” (DEU, 2011, p.20). En tal sentido el DEU reconoce seis tipos de marcas: diatópicas, geográficas, de uso, sociolingüísticas, pragmáticas y técnicas. A su vez, en las sociolingüísticas establece una subdivisión entre registros específicos, valoración social, estratos socioculturales y estilos lingüísticos. Es en atención a todas estas diferencias que elabora las diferentes marcas específicas (urbano, espontáneo, afectivo, hiperbólico, etc.) (DEU, 2011). Las marcas de frecuencia y extensión de uso sirven para descartar, por ejemplo, aquellos términos que ya no son usados, pues si aparecen en el diccionario como poco frecuentes la probabilidad de encontrarlos en un corpus de habla actual es baja.

El DEU no proporciona una justificación de los criterios seguidos para el establecimiento de los distintos tipos de marcas. Para nuestro trabajo, son las de tipo sociolingüísticas y pragmáticas las que resultan más relevantes, dado que es en ellas donde se encuentran las marcas específicas apropiadas para el tratamiento de los insultos. De estas, cabe mencionar las siguientes: desp. (despectivo) y vulg. (vulgar) y más infrecuentemente leng. masc. (lenguaje masculino). Es de suponer que alguna de estas marcas debería acompañar los insultos.

\section{Análisis de los datos: reescritura y discusión}

Lo primero que constatamos, en consonancia con la interpretación de Pullum sobre $\mathrm{AHD}$, es que el DEU no tiene un tratamiento homogéneo de las marcas relativas al tratamiento de los insultos. La razón es que no todas las entradas aparecen con las marcas que ayudan a reconocerlas como tales (muchas no aparecen ni con desp. ni con vulg.). Sin embargo, ello no implica que vengan desprovistas de marca. En tal sentido, hemos consignado, por ejemplo, la aparición de esp. (espontáneo), la que no resulta informativa para los insultos, dado que no diferencia usos despectivos de no despectivos. La misma puede verse en el siguiente ejemplo, donde esp. marca un insulto de grupo relacionado con la indigencia:

(a) Bichicome. (Etim. en inv.) m./f. urb. esp. Persona indigente que vive de desperdicios y pernocta en refugios improvisados $\diamond$ atorrante, ciruja; linyera; piche. // 2. adj. // m./f. urb. esp. desp. machete, - a (1) $\uparrow$ Uso: a veces se emplea la forma bichicoma para el femenino. (DEU, s.v. bichicome).

Ante tales casos, el reconocimiento del insulto demanda otras estrategias. Por ejemplo, podemos o bien recurrir a nuestro conocimiento de la lengua (si es claro que es un insulto para nosotros, aunque el diccionario no lo diga), o bien leer atentamente la entrada para ver si se desprenden de ella indicios de rasgos despectivos 9 .

Si bien el léxico relacionado con insultos de grupo en Uruguay es usado para abarcar múltiples ámbitos, nuestra indagatoria primaria indicaría que el relacionado con las clases sociales es uno de los más productivos y frecuentes en Uruguay. Razón por la cual, pensamos que, para este primer acercamiento al problema, es un buen punto de arranque. En esta oportunidad escogimos tres pares de entradas, el

\footnotetext{
${ }^{9}$ Habíamos pensado, inicialmente, observar los sinónimos que aparecen junto con la definición. La idea era que, si los sinónimos eran insultos, cabría esperar que la entrada también lo fuera. Sin embargo, los sinónimos de un insulto no tienen por qué ser insultos y viceversa (debemos esta puntualización a la Dra. Magdalena Coll, c.p.). De hecho, la tesis que defiende la existencia de un término neutro podría considerarlo como un sinónimo del insulto de grupo y el término neutro no sería un insulto.
} 
primero relacionado con personas indigentes, el segundo con clase social baja y el tercero con la clase social alta.

Veamos con más atención (a) en cuanto a las marcas que acompañan la entrada. Etim. en inv. significa "Etimología en inversión" (viene de "come bichi, bichos"). Es un sustantivo que se usa en masculino y femenino. Es de uso urbano y de lenguaje espontáneo. Llama la atención que no se haya introducido la información despectiva en la entrada. Uno puede entender que le corresponde tal contenido si y solo si se es hablante nativo de un grupo social determinado. Sus sinónimos son, también, despectivos, aunque solo atorrante y piche reciben la marca correspondiente. En la segunda acepción, se presenta otro significado del mismo nombre en el que sí aparece la marca desp. Sin embargo, esta acepción no será analizada al caer fuera de nuestro foco. No es un insulto de grupo, dado que no insulta a un grupo determinado. Insulta a una persona en particular por tener ciertas características. Finalmente, introduce información gramatical suplementaria, en este caso de género.

Si quisiéramos presentar esta entrada mediante el recurso de la metainformación, podríamos reescribirlo así (sin considerar lo referido a los sinónimos $)^{10}$ :

(b) Bichicome m./f. Semántica: indigente que vive de desperdicios y pernocta en refugios improvisados. Metainformación: Es usado informalmente en zonas urbanas de manera despectiva. (s.v. bichicome).

En esta propuesta se restringen las marcas para cubrir la información gramatical, mientras que la información de corte pragmático y/o sociolingüístico es introducida como metainformación léxica.

Veamos un ejemplo del insulto de grupo que es utilizado en Uruguay:

6) me trataron con muy malos modales y como si fuece [sic] un bichicome.

El uso del insulto de grupo en (6) muestra un uso claramente despectivo de bichicome que el hablante entiende es lo que lo hace merecer un tratamiento inadecuado.

Pasemos a la siguiente entrada del DEU:

(c) pichi. (Abr y var. de bichicome). m. / f. // adj. esp. desp. bichicome (1). // 2. m. / f. // adj. esp. desp. Persona sin cualidades apreciables. $\diamond$ pichiruchi. // 3. adj. esp. desp. berreta. // 4. adj. // m. / f. esp. desp. machete, $-a$ (1). // 5. m / f. obs. mil. esp. Persona detenida por la fuerza militar. // 6. m. polic. esp. desp. Malviviente sospechoso de delincuencia. $\diamond$ pia. (DEU, s.v. pichi).

Esta entrada es más sencilla de analizar dado que se presenta como una variante de bichicome, y es esa única acepción la que nos interesa. Las otras acepciones de (c) escapan a nuestro análisis en la medida en que no remiten a clases sociales ni pueden ser clasificadas como insultos de grupo. Lo llamativo de este

\footnotetext{
${ }^{10}$ Aunque los sinónimos comportan información léxica pertinente ésta no ha sido tenida en cuenta en nuestro estudio dado que como señalamos en la nota anterior no acercan la información extralingüística buscada. Es decir, no conllevan la información de que sea un insulto de grupo ni la información de que sea usado despectivamente.
} 
ejemplo es que, aun siendo presentado como variante de bichicome y a diferencia de este, se introduce la marca desp.

En el entendido de que el significado del insulto no puede ser introducido a partir de un sinónimo, proponemos la siguiente reescritura:

(d) pichi. m. / f. // adj. Semántica: indigente que vive de desperdicios y pernocta en refugios improvisados. Metainformación: Es usado informalmente en zonas urbanas de manera despectiva. (s.v. pichi).

El Corpus del Español nos permite recuperar el siguiente ejemplo de pichi usado como insulto de grupo:

7) simplemente es un indigente o un pichi jaja con una graaaaaan rasta sobre su cara.

Al presentar en el mismo contexto el término default indigente y el insulto de grupo pichi (7) muestra mediante una disyunción la posibilidad de que un pichi sea un indigente.

Pasemos al par relativo a clase social baja.

(e) plancha. f. esp. Caída violenta y sorpresiva de una persona. // 2. m. / f. esp. Joven perteneciente a un sector marginal de la sociedad que se caracteriza por una forma peculiar de vestir, de hablar y de relacionarse. // 3. adj. esp. Perteneciente o relativo al modo de ser plancha. // 4. adj. esp. desp. De mal gusto, vulgar. // 5. f. esp. En el fútbol: golpe fuerte, generalmente alevoso, propinado por un jugador a un adversario, con la suela del zapato. // 6. f. Sal. sándwich caliente. // 7. f. polic. Hoja de papel donde aparecen las fotos de quienes han sido detenidos o son buscados por la policía (DEU, s.v. plancha).

Esta larga entrada consigna varios significados de los cuales nos interesa particularmente el segundo. Al igual que lo que ocurría con bichicome no figura la marca desp. Si bien no se desprende de la acepción 2 que sea despectiva, una observación atenta de las acepciones 3 y 4 (claramente relacionadas con ella) nos permite ver el tenor peyorativo que tiene el uso de dicho sustantivo. En este caso, entonces, al recurrir a varias acepciones de la misma entrada podemos reconocer que hay un uso despectivo del término.

Proponemos que sea reescrita de la siguiente manera:

(f) plancha. m. / f. Semántica: Joven perteneciente a un sector marginal de la sociedad que se caracteriza por una forma peculiar de vestir, de hablar y de relacionarse. Metainformación: Usado informalmente de modo despectivo por clases sociales más altas que la implicada. También puede ser usado de manera identificativa y no despectiva por jóvenes pertenecientes a ese grupo (s.v. plancha).

Esta reescritura conjuga las distintas acepciones encontradas en el DEU relacionadas con el insulto de grupo que nos interesa y las integra en una única acepción que permitiría establecer la extensión del término una vez que sea usado en una afirmación. (8) muestra el uso del insulto de grupo y (9) la apropiación que es informada por la metainformación:

8) ...la gente como uno podía comprar Nike. Ahora cualquier plancha tiene uno!!!!

9) Ese acto de orgullo plancha llevó a el error o El Plancha es la Cultura, y vo' lo sabe'. 
Estos ejemplos muestran, claramente, como un mismo término puede ser usado como insulto de grupo, por un lado, y, por el otro, como un uso apropiado por parte de la comunidad/colectividad que está siendo discriminada.

A continuación, analizamos otra entrada relacionada con la clase social baja que permitirá un contraste de modos de introducir información en el DEU.

(g) terraja. m./ f. esp. desp. Persona mal vestida. // 2. m. / f. esp. desp. Persona de condición sociocultural baja. V. pelandrún. // 3. adj. esp. desp. berreta (DEU, s.v. terraja).

La acepción 2 ofrece el insulto de grupo. En ella, además de las marcas gramaticales, se presentan conjuntamente las marcas esp. y desp. notoriamente ausentes en la entrada de plancha. Pese a la presencia de estas marcas, la información semántica brindada en la definición corre el riesgo -probablemente no deseado- de identificar a toda persona proveniente de ese estrato con una persona terraja. Al igual que en el caso de plancha, es la presencia de otras acepciones las que posibilitan una interpretación mejor orientada (ver la 1). Pero a diferencia de este caso, el tipo de información faltante es de tenor semántico y no pragmático o sociolingüístico. No es posible saber, a partir de la entrada anterior, a qué grupo de personas podemos insultar con el uso de dicho término.

Proponemos la siguiente reescritura, que enriquece el contenido semántico de la definición y reubica la otra:

(h) terraja. m. / f. Semántica: Persona de condición sociocultural baja, mal vestida y de gustos cuestionables. Metainformación: Es usado informalmente en zonas urbanas de manera despectiva (s.v. terraja)

A continuación, presentamos un ejemplo de uso del insulto de grupo anterior que muestra, nuevamente, el carácter despectivo y peyorativo que presentan estos insultos en Uruguay:

10) Dios me libre de ese terraja.

Por último, presentamos entradas léxicas de términos despectivos relacionados con la clase social alta:

(i) pituco, - $a$. (¿hipoc. de Petrona?). m. y f. urb. esp. desp. Persona afectada en los modales y en el vestir. // 2. m. y f. / adj. esp. desp. Persona que ostenta una posición social superior. // 3. adj. esp. Referido a una persona: coqueta (DEU, s.v. pituco).

Nuevamente el insulto de grupo corresponde a la segunda acepción. En este caso, al igual que en otros ya vistos, junto a las marcas gramaticales aparecen esp. y desp., pero a diferencia de terraja, la información semántica parecería resultar suficiente. Sin embargo, llama la atención la elección del verbo ostentar (que implica idea de exhibir algo con vanidad y presunción), en la medida que incorpora una valoración negativa a la semántica del término. Por otro lado, al comparar pituco con plancha vemos que la información sociolingüística y pragmática dada 
por las marcas esp. y desp. es completa, por lo que no es necesario recurrir a las otras acepciones contenidas en la entrada.

Proponemos la siguiente reescritura, en la que matizamos la original en cuanto a la ostentación:

(j) pituco, - $-a$. m. y f. / adj. Semántica: Persona que exhibe una posición social superior. Metainformación: Es usado informalmente de manera despectiva (s.v. pituco).

La reescritura propuesta permite explicar por qué en el próximo ejemplo es evidente la despectividad.

11) Y a quien pondrían de Alcalde de Punta? Tiene que ser un pituco de estos!! Se dan un baño de Pueblo (como dijo una vez Paez Vilaró) y ta... tenemos un pituco remasterizado apto para entrar a el barrio más jodido de todos.

La última entrada que analizaremos es un insulto de grupo referido a la misma clase social.

(k) cheto, - a (Afér. de concheto). adj. esp. referido a una cosa: distinguida o selecta. // 2. m. y f. / adj. esp. desp. Persona que pertenece o aparenta pertenecer a una clase social acomodada. $\diamond \diamond$ concheto. (DEU, s.v. cheto).

Una vez más el insulto de grupo corresponde a la segunda acepción. Al igual que en el caso anterior, las marcas cubren tanto la información gramatical como la sociolingüística y pragmática. El problema es determinar si la información semántica es suficiente o no. Parece ser que, al introducir "aparenta pertenecer", la información dada por el diccionario nos induce a pensar que se debe cumplir con ciertos requisitos, probablemente de vestimenta, para que alguien pueda ser insultado con la palabra cheto.

Proponemos la siguiente reescritura:

(1) cheto, - $a$. m. y f. / adj. Semántica: Persona que pertenece o aparenta pertenecer a una clase social acomodada. Metainformación: Es usado informalmente de manera despectiva (s.v. cheto)

En el ejemplo siguiente se nota claramente un tenor despectivo y condescendiente en el uso del insulto de grupo anterior:

12) Y a quien pondrían de Alcalde de Punta? Tiene que ser un pituco de estos!! Se dan un baño de Pueblo (como dijo una vez Paez Vilaró) y ta... tenemos un pituco remasterizado apto para entrar a el barrio más jodido de todos.

\section{Consideraciones finales}

El estudio de los insultos de grupo es un campo abierto al interés de múltiples disciplinas -filosofía del lenguaje, ética y metaética, psicología, lingüística, etc.-, razón por la cual aproximaciones lingüístico-lexicográficas resultan fundamentales. Este trabajo en proceso intenta hacer un aporte en dicha dirección, al considerar tanto aspectos lingüísticos como desarrollos teórico-filosóficos.

Al analizar las diversas entradas léxicas pudimos observar que las definiciones dadas por el DEU no son homogéneas: algunas proporcionan información semántica suficiente para determinar a quiénes se aplican los términos, pero no la información pragmática o sociolingüística -la metainformaciónsuficiente, y viceversa. Encontramos, a su vez, que el diccionario describe en mayor 
profundidad términos más recientes, como plancha, y en menor profundidad términos como terraja cuya definición parece insuficiente para poder determinar el grupo social al que se aplica dicho insulto.

Creemos que las reescrituras que hemos propuesto para cada entrada cuentan con la ventaja de eliminar de las definiciones los contenidos que no pertenecen a la semántica del léxico. Por otra parte, al proporcionar la metainformación, presentan elementos necesarios para que el usuario del diccionario pueda saber que dichos términos son utilizados para discriminar a individuos que pertenecen a una clase social determinada.

Queremos cerrar afirmando que no es intención de este trabajo el problematizar la noción de insulto de grupo, ni tampoco problematizar sobre las decisiones tomadas por los lexicógrafos al elaborar el DEU.

Nuestro interés, en cambio, ha consistido en indagar cómo se podría ajustar un diccionario de nuestra variedad para dar cuenta de la idea de Pullum de que para una presentación lexicográfica que refleje adecuadamente el contenido de los insultos de grupo se requiere la incorporación de información adicional a la propiamente semántica. Al hacerlo, pudimos obtener una primera aproximación al estudio lexicográfico de los insultos de grupo en Uruguay.

\section{Referencias}

1. Academia Nacional de Letras (2011). Diccionario del Español del Uruguay. Montevideo: Banda Oriental. [Cit. como DEU (2011)].

2. Anderson, L., y Lepore, E. (2013). What did you call me? Slurs as prohibited words. Analytic Philosophy, 54(3), pp. 350-363. DOI: https://doi.org/10.1111/phib.12023

3. Cepollaro, B. (2017) Slurs as the Shortcut of Discrimination. Rivista di estetica, 64, pp. 53-65. DOI: https://doi.org/10.4000/estetica.2063

4. Cepollaro, B. y Zeman, D. (2020) Editors' Introduction. The Challenge from Non-Derogatory Uses of Slurs. Grazer Philosophische Studien, 97, pp. 1-10. DOI: https://doi.org/10.1163/18756735-09701002

5. Croom, A. (2013). How to do things with slurs: Studies in the way of derogatory words. Language \& Communication, 33, pp. 177-204. DOI: https://doi.org/10.1016/j.langcom.2013.03.008

6. DiFranco, R. (2015). Do racists speak truly?, Thought, 4, pp. 28-37. DOI: https://doi.org/10.1002/tht3.154.

7. Grice, H. P. (1975). Logic and Conversation. En Cole, P., y Morgan J. (Ed.), Syntax and Semantics. San Diego: Academic Press. DOI: https://doi.org/10.1163/9789004368811_003.

8. Hom, C. (2012). A Puzzle about Pejoratives. Philosophical Studies, 159, pp. 383-405. DOI: https://doi.org/10.1007/s11098-011-9749-7

9. The American Heritage Dictionary of the English Language. Houghton Mifflin Harcourt Publishing. Recuperado de: https://ahdictionary.com/.

10. Maitra, I. y McGowan, K., (Eds.) (2012). Speech and Harm. Controversies over Free Speech. Oxford: Oxford University Press. DOI: https://doi.org/10.1093/acprof:oso/9780199236282.001.0001

11. Matsuda, M. J. (1993). Words That Wound: Critical Race Theory, Assaultive Speech, and the First Amendment (New Perspectives on 
Law, Culture, and Society), Boulder, Colorado: Westview Press.

12. McCready E. (2010). Varieties of Conventional Implicature.

Semantics and Pragmatics, 3(8), pp. 1-57. DOI:

https://doi.org/10.3765/sp.3.8

13. Neufeld, E. (2019). An Essentialist Theory of the Meaning of Slurs.

Philosophers' Imprint, 19(35). Recuperado de:

https://philpapers.org/archive/NEUAET.pdf

14. Nunberg, G. (2018). The social life of slurs. En D.Fogal, D.W. Harris y M. Moss, (Eds.), New work on speech acts (pp. 237-295). Oxford:

Oxford University Press. DOI:

https://doi.org/10.1093/oso/9780198738831.003.0010

15. Oggiani, C. y Bértola, C. (2019). Elatividad en el español del Uruguay. Recategorización de propio/a. En Riestra D., y Múgica N. (Ed.), Estudios SAEL 2019 (pp. 59-74). Bahía Blanca: Ediuns y Sael.

16. Orlando, E. y Saab, A. (2019). Términos peyorativos de grupo, estereotipos y actos de habla. Crítica. Revista Hispanoamericana de Filosofía, 51(153).

17. Orlando, E. y Saab. A. (en prensa). Epítetos e insultos de grupo en español. Sobre una ambigüedad y sus implicaciones sintácticosemánticas. Aceptado para publicación en: Studies in Hispanic and Lusophone Linguistics.

18. Potts, C. (2005). The Logic of Conventional Implicatures. Oxford: Oxford University Press. DOI:

https://doi.org/10.1093/acprof:oso/9780199273829.001.0001

19. Pullum, G. K. (2018). Slurs and obscenities: lexicography, semantics, and philosophy. En Sosa, D. (Ed.), Bad Words. Philosophical Perspectives On Slurs (pp. 168-192). Oxford: Oxford University Press. DOI: https://doi.org/10.1093/oso/9780198758655.003.0009

20. Russel, B. (1905). On denoting. Mind, 14, pp. 479-493. DOI: https://doi.org/10.1093/mind/XIV.4.479

21. Sosa, D. (2018). Introduction. En Sosa D. (Ed.), Bad Words. Philosophical Perspectives On Slurs (pp. 1-5). Oxford: Oxford University Press. DOI:

https://doi.org/10.1093/oso/9780198758655.001.0001

22. Whiting, D. (2013). It's Not What You Said, It's the Way You Said It: Slurs and Conventional Implicature. Analytic Philosophy, 54(3), pp. 364-367. DOI: https://doi.org/10.1111/phib.12024

23. Williamson, T. (2009). Reference, Inference and the Semantics of Pejoratives. En Almog, J., y Leonardi P. (Ed.), The Philosophy of David Kaplan (pp. 137-158). Oxford: Oxford University Press. DOI: https://doi.org/10.1093/acprof:oso/9780195367881.003.0009 